\title{
Recent advances of DNAzyme-based nanotherapeutic platform in cancer gene therapy
}

\author{
Wendi Huo ${ }^{1}$, Xiaona $\mathrm{Li}^{2}$, Bei Wang ${ }^{1}$, Haoran Zhang ${ }^{1}$, Jinchao Zhang ${ }^{2}$, \\ Xinjian Yang ${ }^{2 \bowtie}$, Yi Jin ${ }^{1 凶}$ \\ ${ }^{1}$ College of Basic Medical Science, Key Laboratory of Medicinal Chemistry and Molecular Diagnosis of the Ministry \\ of Education, Key Laboratory of Pathogenesis Mechanism and Control of Inflammatory-autoimmune Diseases of \\ Hebei Province, Hebei University, Baoding 071002, Hebei, China \\ ${ }^{2}$ College of Chemistry \& Environmental Science, Key Laboratory of Medicinal Chemistry and Molecular Diagnosis of \\ the Ministry of Education, Chemical Biology Key Laboratory of Hebei Province, Institute of Life Science and Green \\ Development, Hebei University, Baoding 071002, Hebei, China
}

Received: 26 June 2020 / Accepted: 8 October 2020 / Published online: 20 November 2020

\begin{abstract}
Deoxyribozyme (or denoted as DNAzyme), which is produced by in vitro screening technology, has gained extensive research interest in the field of biomedicine due to its high catalytic activity and structure identification. This review introduces the structural characteristics of RNA-cleaving DNAzyme and its application potential in cancer gene therapy, which plays a significant role in cancer-related gene inactivation by specifically cleaving target mRNA and inhibiting the expression of the corresponding protein. However, the low delivery efficiency and cellular uptake hindered the widespread usage of DNAzyme in gene therapy of cancers. Emerging nanotechnology holds great promise for DNAzyme to overcome these obstacles. This review mainly focuses on DNAzyme-based nanotherapeutic platforms in gene therapy of cancers, including oncogene antagonism therapy, treatment resistance gene therapy, immunogene therapy, and antiangiogenesis gene therapy. We also revealed the potential of DNAzymebased nanotherapeutic platforms as emerging cancer therapy approaches and their security issues.
\end{abstract}

Keywords DNAzyme, Cancer therapy, Gene-silencing, Nanocarrier

\section{INTRODUCTION}

DNAzymes have been demonstrated to specifically perform the functions of the degradation of target mRNA, ligation, or phosphorylation of DNA, and so on. They are obtained by in vitro screening technology and show high structure recognition ability (Fu and Sun 2015; Hollenstein 2015). Since the first proposition in 1994 by Breaker, various DNAzymes have been discovered. Among these DNAzymes, the one with RNAcleaving activity has attracted extensive

Wendi Huo and Xiaona Li have contributed equally to this work.

$\bowtie$ Correspondence: jianxinyang123@163.com (X. Yang), jinyi2009@163.com (Y. Jin) research interests due to its great potential in cancer therapy (Zhou et al. 2017). After the catalytic cleaving target mRNA, the expression of corresponding proteins is then downregulated. To realize the gene-silencing, the structure of the DNAzyme should be integrated. Each DNAzyme has a core catalytic zone which flanked by two-side recognition arms. The longer the recognition arms, the more stable the DNAzyme binds to its substrates by Watson-Crick basis pairing (Dass et al. 2008; Fokina et al. 2012; Santoro and Joyce 1997; Silverman 2005). In this way, the special sequence of recognition arms determines the specificity of the DNAzyme and stabilizes the catalytic core of the DNAzyme to realize the hydrolysis reaction (Khachigian 2019). Of all types of DNAzymes, 10-23 DNAzyme is the most popular one 
because it can cleave almost any phosphodiester bond between the unpaired purines and the paired pyrimidine (Xu et al. 2012). In the presence of specific metal ions such as $\mathrm{Mg}^{2+}$ (Cao et al. 2020), $\mathrm{Pb}^{2+}$ (Lan et al. 2010), $\mathrm{Mn}^{2+}$ (Wang et al. 2019a), $\mathrm{Cu}^{2+}$ (Liu and $\mathrm{Li}$ 2007), and $\mathrm{Na}^{+}$(Zhou et al. 2016), DNAzyme can successfully cut off the target mRNA by catalyzing the hydrolysis of the phosphodiester of target RNA (Fig. 1). Therefore, DNAzyme may become a potential and powerful tool for gene-regulating and provide a novel gene therapy approach for cancer therapy.

As an important tool of gene therapy, DNAzyme shows some inherent advantages compared with other enzymes. Firstly, DNAzyme has unparalleled structural stability than protein and ribozyme. Secondly, it can cut target mRNA more accurately than the ribozyme without any immune responses and significant cytotoxicity. Furthermore, DNAzyme has the characteristics of high specificity, easy modification and functionalization, and low cost (Li et al. 2018; Ren et al. 2019; Silverman 2016). Theoretically, the DNAzymes can be used to silence any genes as we expect which are preferable for cancer treatment. The specific catalytic activity of DNAzymes makes them applicable for precise gene therapy. Following the study of bcr-abl oncogene-targeted DNAzyme for acute lymphoblastic leukemia, DNAzyme acts as a novel class of biotechnology-derived drugs and has shown great significance in the field of cancer gene therapy (Wu et al. 1999). Although some breakthroughs have been made, there are still some unavoidable challenges in applying DNAzyme in cancer treatment. The first key issue is how to efficiently deliver DNAzymes to cancer cells. DNAzymes have to overcome multiple physiological barriers during transportation from the administration site to cancer tissues, including high interstitial pressure of the tumor tissue, the abundant tumor extracelllular matrix (ECM), and high risk of being degraded by nucleases (Farrokhi et al. 2018;

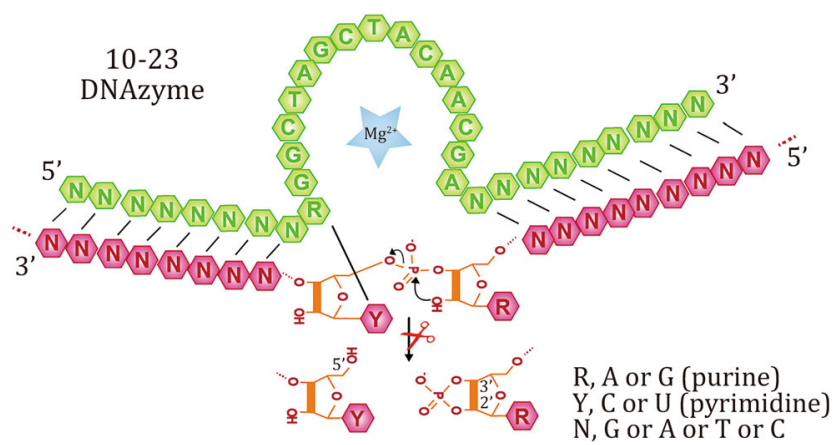

Fig. 1 The action mechanism of DNAzyme with RNA-cleaving activity
Xiao et al. 2019). Therefore, it is necessary to explore stable vectors to improve the efficiency of DNAzymes for overcoming these obstacles. Another key issue is that the cofactors in organisms are insufficient to support the catalytic activity of DNAzymes, which reduces their gene-silencing efficiency (Feng et al. 2018; Roma-Rodrigues et al. 2020).

In recent years, emerging nanomedicine brings new opportunities for cancer gene therapy. The nanocarriers with a proper size can prevent DNAzymes from degradation and deliver the DNAzymes to tumor cells after intravenous administration by "enhanced permeability and retention (EPR) effect" and positive targeting (Davis et al. 2008; Maeda et al. 2000). Thus, a nanotherapeutic platform is an effective means to improve the stability, catalytic capacity, and the delivery efficiency of DNAzymes (Roma-Rodrigues et al. 2020; Xiao et al. 2019). In this review, we summarized recent progress in the development of DNAzyme-based nanoplatform for oncogene antagonism, reversing therapeutic resistance, immunogene therapy, and antiangiogenesis in gene therapy of cancers and made an outlook on their clinical application in the future.

\section{THE APPLICATION OF DNAZYME-BASED NANOPLATFORMS IN CANCER GENE THERAPY}

Cancer progression is a complex and multi-step process, which involves the mutation or abnormal expression and regulation of multiple cancerassociated genes including activation of oncogenes, high expression of apoptosis suppressor genes, and drugresistant genes in tumor cells, and so on. A series of gene therapy strategies have been designed to target these cancer-associated genes, such as oncogene antagonistic therapy, antiangiogenic therapy, immune gene therapy, and drug-resistant gene therapy. Based on the remarkable advantages of DNAzyme, gene intervention strategy using an RNA-cleaving DNAzyme has been one promising alternative for cancer therapy. Recent progress on DNAzyme focuses on how to develop smart nanoplatforms for efficiently delivering DNAzyme for cancer gene therapy.

\section{DNAzyme-based nanoplatforms for oncogene antagonism}

More and more studies show that both oncogenes and antioncogenes play key roles in regulating the proliferation and metastasis of tumor cells. In general, various stimuli including virus infection, chemical carcinogens, and radiation can activate the oncogenes to 
promote the progression of cancers. Therefore, the oncogenes can be used as ideal targets for DNAzymes to enhance the therapeutic effect.

Unlimited proliferation is one characteristic that distinguishes cancer cells from normal cells. C-jun is a member of the activating protein 1 (AP-1) transcription factors that participate in cellular proliferation, transformation, and apoptosis. It was reported that the overexpression of c-jun in cancer cells could increase the activity of AP-1, which was closely related to the highly invasive property of malignant cancers (Peng et al. 2016). Tan et al. established a c-jun mRNA-targeting DNAzyme (Dz13) enfolded chitosan nanoparticles (Dz13-NP) with a size of $50-300 \mathrm{~nm}$. The in vivo and in virto results demonstrated the high stability of Dz13-NP as well as the activity of cleaving c-jun mRNA thus inhibiting tumor growth (Tan et al. 2010). Meng et al. demonstrated the effectiveness and feasibility of DNA tetrahedron as DNAzyme nanocarriers for gene-silencing therapy. These nanostructures have high cellular uptake ability to downregulate the c-jun mRNA, resulting in the inhibition of cell proliferation (Fig. 2) (Meng et al. 2019). The miR21 is an endogenous non-coding short single-stranded RNA, and emerging evidence shows that the overexpression of miR-21 is related to the proliferation and invasion of cancer cells. Recently, Ren et al. demonstrated that DNA tetrahedron could be used to co-delivery (antimiR-21) 17E DNAzyme, ZY11-targeting aptamer, and doxorubicin (DOX) for synergistic cancer therapy (Ren et al. 2019). This nanoplatform shows favorable tumor targeting and $\mathrm{pH}$ responsiveness property to achieve remarkable gene-silencing efficiency. The results indicated that the DNA tetrahedron was a promising delivery nanocarrier for tumor-targeted multimodal therapy. In addition, a study by Wang and coworkers reported a DNA methyltransferase (DNMTs)-specific DNAzyme which was used to significantly suppress tumor growth in the model of bladder cancer mouse (Wang et al. 2015). Li et al. designed a multifunctional DNA nanoscorpion functioned with specific aptamers and human epidermal growth factor receptor (HER2) mRNA-targeting DNAzyme. The results displayed that the DNA nanoscorpion had the capability of efficiently targeting and downregulating specific targets to suppress tumor growth (Li et al. 2018). To enhance the catalytic efficiency of DNAzyme due to the lack of cofactors, a study by He and his colleagues revealed the availability of in situ cofactor generation strategy for gene-silencing therapy ( $\mathrm{He}$ et al. 2016). They exploited a dual-functional nanocomposite equipped with gold nanoparticles (GNPs), AS1411 aptamer, anti-miR-21 DNAzyme, DOX, and acid-decomposable $\mathrm{ZnO}$ quantum dots as a self-generation pool of $\mathrm{Zn}^{2+}$ ions, to realize the high efficiency of intracellular genesilencing. Similarly, Wang et al. synthesized DNAzyme nanosponges (NSs) which were composed of multivalent tandem aptamer sequences, DNAzymes, ZnO NPs, and DOX. The NSs collapsed in the acidic condition to generate $\mathrm{Zn}^{2+}$, which acted as not only the DNAzyme cofactors to mediate the self-catabolic activity of DNAzyme, but also the therapeutic reactive oxygen species generators. This novel and intelligent self-driven drug delivery system might be engineered with more therapeutic agents and shows great promise and versatility for biomedicine applications. (Wang et al. 2019b). In addition, nanoscaled zeolitic imidazolate framework-8 (ZIF-8) NPs, which collapsed in the acid conditions to generate $\mathrm{Zn}^{2+}$, were used as delivery vehicles of DNAzymes to overcome the limitation meditated by insufficient of cofactors and promote the early growth response-1 (EGR-1)-associated antiproliferation efficacy (Wang et al. 2019a). These smart nanocarriers not only protect DNAzymes from digestion by endogenous nucleases, but also increase the targeted oncogenes silencing activity of DNAzyme in the cytoplasm, leading to enhanced antiproliferation effect.
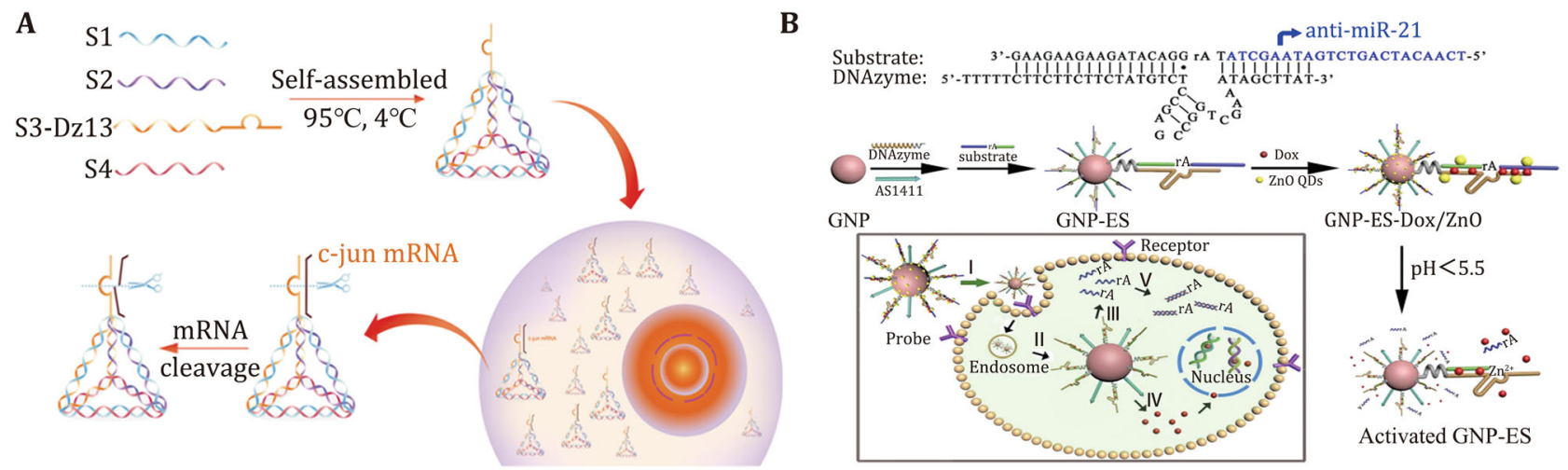

Fig. 2 A The self-assembled TDNs modified with a c-jun targeting DNAzyme realize higher cellular uptaken efficiency and gene-silencing activity (Meng et al. 2019). B A multifunction nanocomposite probe consisted of GNPs, DNAzyme, Dox, AS1411 aptamer, and ZnO QDs for achieving intracellular gene regulation and anticancer drug delivery (He et al. 2016) 
Apoptosis has an essential role in the occurrence, progression, and metastasis of cancers. In most cancers, the inhibitors of apoptosis proteins (IAP) that contribute to tumor cells apoptosis resistance are overexpressed. Therefore, the development of nanotherapeutic platforms targeting IAP has attracted much attention. Survivin is a powerful antiapoptotic gene and overexpressed in most cancers. For the last few years, our laboratory has been working on developing DNAzyme-based multifunctional nanocarriers that target apoptosis-related genes to enhance cancer therapy outcomes. Recently, we synthesized a biodegradable, dual-target nanoflowers (DNFs) functionalized with antisurvivin DNAzyme, anti-EGR-1 DNAzyme, and AS1411 aptamer using rolling circle amplification (RCA) strategy. The DNFs degrade at acidic condition due to the decomposition of the coassembled magnesium pyrophosphate framework, generating abundant $\mathrm{Mg}^{2+}$. The additive $\mathrm{Mg}^{2+}$ could serve as the cofactor of the DNAzyme to enhance its efficiency to cleave apoptosis-related target mRNAs (Fig. 3). We predicted that this biodegradable, multifunctional therapeutic system has great potential for high efficiency gene therapy (Jin et al. 2017). In addition, antisurvivin DNAzyme could also be used to enhance the efficiency of photodynamic therapy (PDT). We found that the upconversion nanoparticles (UCNPs) could work as energy donors of photosensitizers $\left(\mathrm{TMPyP}_{4}\right)$ to activate the PDT in the presence of NIR light. However, the overexpressed antiapoptotic proteins would increase the difficulty of PDT treatment. In this work, the loaded DNAzymes could efficiently silence the survivin gene and amplify the therapeutic outcome. As a result, this nanoplatform would be used as an admirable alternative to enhance the efficiency of PDT with the help of adjuvant gene therapy (Jin et al. 2020). These studies demonstrated the feasibility and effectiveness of the IAP targeting DNAzymes to synergistically enhance therapeutic performance. EGR-1 is another key factor in regulating cell proliferation, differentiation, and apoptosis of malignant tumors. Wang et al. employed $\mathrm{MnO}_{2}$ nanosheets to deliver EGR-1 DNAzymes and photosensitizers toluidine blue (TB) (Wang et al. 2019c). The $\mathrm{MnO}_{2}$ nanosheets were employed as both delivery carriers and the pool of DNAzyme cofactor due to their appealing physicochemical properties. The $\mathrm{MnO}_{2}$ nanosheets were degraded by the intracellular GSH to produce $\mathrm{Mn}^{2+}$, which served as cofactors of DNAzyme to enhance the silencing efficiency. Meanwhile, the
A

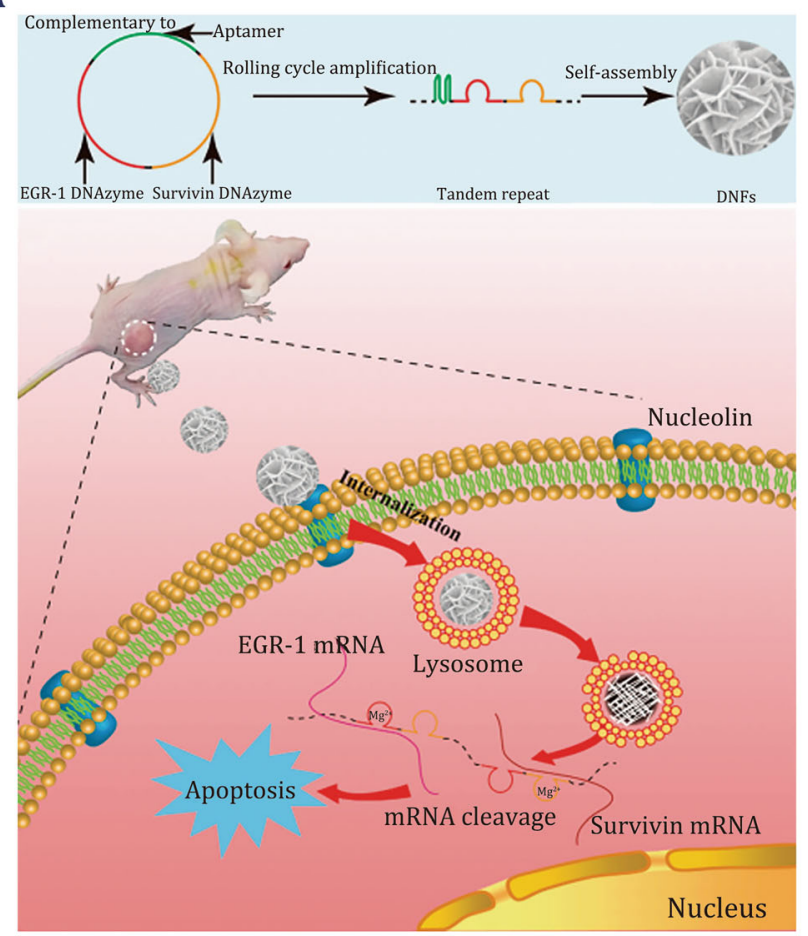

B

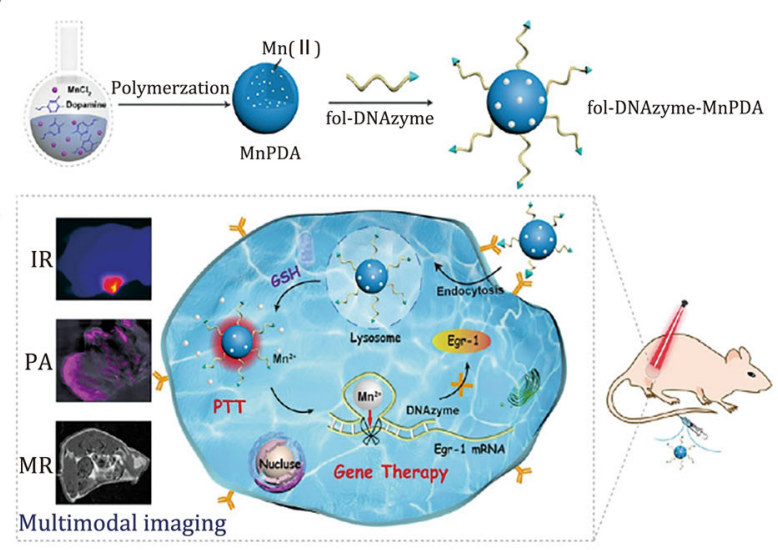

C

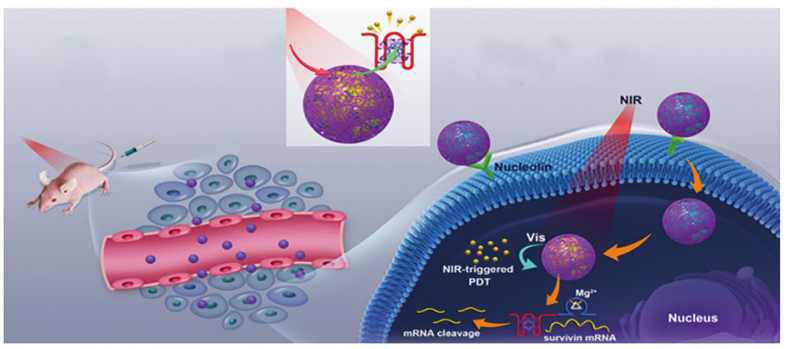

Fig. 3 DNAzyme-based nanotherapeutic platform in oncogene antagonistic therapy. A The DNFs target the cancer cell to achieve efficient dual gene-silencing (Jin et al. 2017). B The fol-DNAzyme-MnPDA nanoplatform as a versatile vehicle for multimodal imaging-guided gene regulation and photothermal therapy. (Feng et al. 2018). C The multifunctional DNAzyme-assisted upconversion nanoplatform for enhanced PDT by DNAzyme-mediated gene-silencing of survivin (Jin et al. 2020) 
consumption of GSH further improved the PDT efficiency. Similarly, the Ce6-DNAzyme- $\mathrm{MnO}_{2}$ nanosystem was synthesized by Fan et al. to overcome the limitation stemmed from the inefficiency and poor biostability of DNAzyme (Fan et al. 2015). Apart from these researches, a recent study by Feng et al. utilized the polydopamine- $\mathrm{Mn}^{2+}$ nanoparticles (MnPDA) as the vehicles of EGR-1 targeting DNAzyme (Feng et al. 2018). Results showed that the MnPDA not only downregulated the EGR-1 mRNA efficiently but also served as magnetic resonance imaging (MRI) contrast agents, thus realizing the integration of tumor detection and treatment.

Metastasis, as a prominent feature of malignant tumors, is a major factor affecting prognosis. Silencing metastasis-related mRNA will attenuate the probability of tumor metastasis. Xing et al. used the N-acetyl-Lleucine-polyethylenimine (N-Ac-L-Leu-PEI) as the DNAzyme vectors to targeted silence aurora kinase A mRNA. The nanocomplex presented excellent cellular uptake ability and great potential in cleaving target mRNA, which would induce apoptosis and inhibit tumor metastasis (Xing et al. 2015). Integrin is a member of cell adhesion molecules (CAM) which affects the growth, proliferation, and invasion of tumors through meditating the interaction between cancer cells and the ECM. Wiktorska et al. employed liposomes to transfect $\beta 1$ targeting DNAzyme for specifically cleaving $\beta 1$ integrin mRNA and eradicating the invasive cancer cells (Wiktorska et al. 2010). All these reports clearly demonstrated the effectiveness of DNAzyme in attenuating the probability of tumor metastasis.

\section{DNAzyme-based nanoplatforms for treatment resistance}

Treatment resistance, whether inherent or acquired, is a major problem leading to the failure of cancer therapy. Crafty cancer cells have evolved various resistance mechanisms that protect them from being killed by chemotherapy drugs, heat, and radiation. For example, the blockage of the apoptosis pathway, the overexpression of multidrug resistance-related proteins, and the stress protective function of cancer cells contribute to treatment resistance (Ramos and Bentires-Alj 2015). Therefore, it is necessary to develop effective approaches to restore the sensitivity of cancer cells to various treatments, which might provide new therapeutic options for cancer therapy. In this respect, our laboratory has done some work on developing multifunctional nanoplatforms for overcoming the therapeutic resistance. We synthesized a DNAzyme nanosponges by assembling a cationic polymer polyetherimide (PEI) and a long single-stranded DNA encoded with multivalent DNAzymes for avoiding thermo-resistance in photothermal therapy (PTT). The results showed the capability of these nanosponges to downregulate the heat shock protein 70 (HSP70) mRNA for reversing the resistance of cancer cells to hyperthermia (Fig. 4) (Jin et al. 2018). Due to its simple synthesis, good biocompatibility, and high efficiency, nanosponge-ICG is a promising therapeutic drug for PTT. Triple-negative breast cancer (TNBC) is the most difficult type of breast cancer to treat due to its severe therapeutic resistance to chemotherapy or radiotherapy. To increase the sensitization of TNBC cells to chemotherapy drugs, we constructed a NIR-light activated combination therapeutic nanoplatform using mesoporous silica-coated gold nanorods (Au@MSNs) modified with survivin targeting DNAzyme. Once exposed to NIR-light irradiation, the absorbed NIR light by gold nanorods was converted into heat, which triggered the release of DNAzyme to downregulate survivin mRNA for sensitizing MDA-MB231 cells, resulting in enhanced PTT effect (Sun et al. 2018).

Multidrug resistance (MDR) has been the primary obstacle that contributes to the failure of chemotherapy. It was reported that more than $90 \%$ of the death with malignant tumors are related to MDR. Up to now, a variety of drug resistance-related factors have been discovered. For example, the multidrug resistance gene 1 (MDR1) encodes the plasma membrane P-glycoprotein (P-gp), which is a member of the ATP-binding cassette (ABC) transporter, functions as the energy-dependent "drug pump" to flush out the lipophilic chemotherapy drugs, resulting in the decrease of intracellular drugs (Gao et al. 2006). Zokaei et al. synthesized an MDR1-targeting DNAzyme delivery platform using chitosan $\beta$-cyclodextrin nanocomplexes. After the silencing of the MDR1 gene, DOX-resistant breast cancer cells MCF-7/DR showed enhanced sensitivity toward DOX, achieving a high antitumor therapy effect (Zokaei et al. 2019). In addition, MDR can also be reversed by reducing nonMDR-associated proteins, such as pro-apoptotic c-jun protein. Sun et al. used mesoporous silica nanoparticles (MSNs) to deliver c-jun targeting DNAzyme to attenuate the drug efflux because silencing c-jun mRNA mediated the downregulation of multidrug resistance-associated protein 1 (MRP1), which is bound up with the acquired MDR in metastatic prostate cancer (Sun et al. 2017). Dass et al. also indicated the role of DNAzymes-mediated silencing c-jun gene in reversing the drug resistance of osteosarcoma. The in vitro experiments demonstrated its role in restoring the chemosensitivity as well as 
A
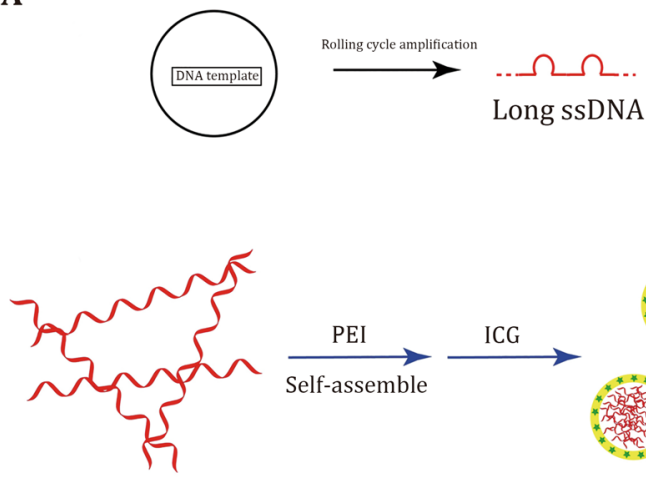

Long ssDNA

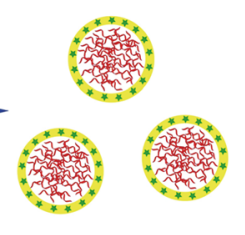

Nanosponge-ICG
B

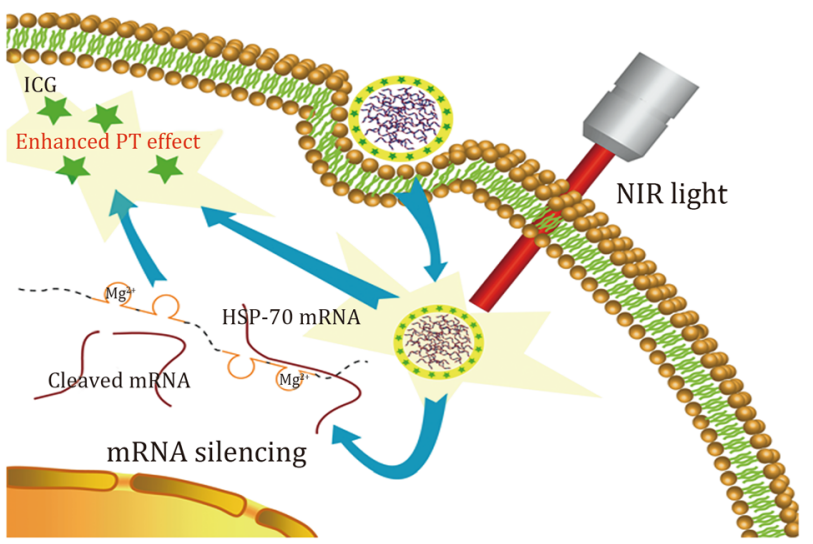

Fig. 4 The synthesis of DNAzyme-based nanosponges (A) and enhanced PT effect by silencing HSP70 mRNA (B) (Jin et al. 2018)

inhibiting tumor metastasis (Dass et al. 2008). Bcl-xl has also been demonstrated to be associated with apoptosis and drug resistance (Amundson et al. 2000). Yu et al. developed a Bcl-xl targeting DNAzyme system to restore the sensitivity of the drug resistance cells to taxol (Yu et al. 2014). Although treatment resistance is an intractable issue that could not be completely avoided, DNAzymes show great potential in silencing treatment resistance-related mRNA to recover the sensitivity of cancer cells. All the above reports revealed the excellent efficiency of DNAzymebased nanoplatforms in enhancing the overall therapeutic effect.

\section{DNAzyme-based nanoplatform for immunogene therapy}

Cell-mediated adaptive immunity plays an important role in the antitumor immune response. T lymphocytes mainly consist of $\mathrm{CDB}^{+}$cytotoxic $\mathrm{T}$ cells and effector $\mathrm{CD}^{+}{ }^{+} \mathrm{T}$ cells. $\mathrm{CD} 8^{+}$cytotoxic $\mathrm{T}$ cells can be activated by the stimulus signals from dendritic cells (DCs) and $\mathrm{CD} 4{ }^{+} \mathrm{T}$ cells, directly killing tumor cells, while $\mathrm{CD} 4^{+} \mathrm{T}$ cells have a role in the proliferation and activation of $\mathrm{CD}^{+}$cytotoxic T cells and the establishment of memory. Meanwhile, $\mathrm{CD} 4^{+} \mathrm{T}$ cells can not only kill tumor cells by the cytokines that they secrete, such as TNF- $\alpha$, but also directly destroy tumors by recognizing major histocompatibility complex (MHC) molecules on the surface of tumor cells (Raphael et al. 2020; Haabeth et al. 2014). Therefore, selectively silencing specific tumor-related genes by DNAzyme can be used to directly or indirectly activate the adaptive immune of the body for enhanced antitumor effect.

Previous researches have demonstrated that Dz13 could induce adaptive immunity (Khachigian et al. 2012). The preclinical safety and in vivo efficiency of
Dz13 were confirmed in various tumor-bearing mice. Subsequently, the safety and tolerability of Dz13 in human beings were assessed in phase 1 clinical study in 2013. The results demonstrated that the tumor regressed significantly without any side effect after the intratumoral administration of DNAzyme-loaded lipid. Immunohistochemical staining showed that the antitumor effect was ascribed to the increased expression of apoptosis markers caspases 3, 8, 9, and P53, and the decreased expression of Bcl-2 and MMP-9. Meanwhile, the expression of CD4 and CD8, markers of T-lymphocyte infiltration into basal-cell carcinoma, was increased, indicating stimulated infiltration of immune cells (Cho et al. 2013). Cai et al. demonstrated that Dz13 could induce adaptive immunity in skin cancers. The DNAzyme increased the content of $\mathrm{CD}^{+}$and $\mathrm{CD} 4^{+} \mathrm{T}$ cells three-to-four-fold in immunocompetent mice, resulting in enhanced antitumor immunity, which contributes to the enhanced antitumor effect (Cai et al. 2012). In subsequent work, they tested the antitumor immunotherapy effect of a c-jun targeting DNAzyme. They demonstrated the capability of DNAzyme-loaded lipid to downregulate the expression of c-jun and increase the content of $\mathrm{CD} 4^{+} \mathrm{T}$ cells in the tumors. Thus, the DNAzyme-loaded lipid can effectively inhibit B16F10 tumor growth and abscopal tumor relapse through adaptive immunity (Cai et al. 2018). To improve the intracellular delivery efficiency of DNAzyme, Peng et al. used nanosize metal-organic frameworks (MOFs) to deliver DNAyzme into RAW264.7, $\mathrm{CD}^{+}{ }^{+} \mathrm{T}$ cells, and B cells. These MOFs not only exhibit excellent transfection efficiency in mammalian immune cells than conventional transfection agents, but also reversibly uptake and release DNAzyme in cancer cells, and protect them from digestion by nuclease (Peng et al. 2018). In addition, innate immunity also plays a role in defending against cancer. Jang et al. 
developed a treatment platform containing DNAzyme and $\mathrm{CpG}$ sequence to simultaneous silence epidermal growth factor receptor (EGFR) to promote the apoptotic cell death and activate Toll-like receptor 9 (TLR9)dependent p38 MAPK signaling pathway via the innate immune response and resulted in a stronger inhibition of non-small-cell lung cancer proliferation (Jang et al. 2018). Compared with traditional antibody therapy and adoptive cell therapy, DNAzyme-based immunogene therapy is more economical and safe. Therefore, the DNAzyme-based nanoplatforms provide a new approach for tumor immunotherapy.

\section{DNAzyme-based nanoplatform for antiangiogenesis}

Angiogenesis plays a crucial role in the growth and metastasis of malignant tumors (Cully 2017). The newly formed blood vessels could transport plentiful oxygen and nutrients deep into the tumor to promote the rapid proliferation of tumor cells. Thus, the antiangiogenesis strategy is determined to be a promising approach for blocking the progression of malignant tumors. At present, the antiangiogenic drugs approved by the U.S. FDA show serious side effects. Therefore, it is necessary to develop more safe and effective antiangiogenic adjuvant therapy strategies. Antiangiogenesis gene therapy strategies involve the expression inhibition of proangiogenic molecules, as well as the apoptosis induction of vascular endothelial cells (Lugano et al. 2020).

As we know, tumor angiogenesis involves multiple signaling molecules, such as VEGF, VEGFRs, and matrix metal proteinases (Goel and Mercurio 2013). Many researchers have reported that the inhibition of angiogenesis could be realized by the downregulation of VEGF or its tyrosine kinase receptor through DNAzymebased gene-silencing. Zhang et al. used transcatheter arterial chemoembolization (TACE) technology to efficiently deliver the VEGFR-1 targeting DNAzyme into tumors. Results showed that the expression level of VEGFR-1 mRNA in rabbit corneal endothelial cells and tumor tissue decreased. After treating with DNAzyme, the permeability of tumor microvascular was suppressed through non-invasive dynamic contrast enhanced magnetic resonance imaging (DCE-MRI) (Zhang et al. 2016). Shen et al. designed a VEGFR-1 mRNA-targeting DNAzymes, which could efficiently inhibit the VEGFR-1 expression, tube formation, and tumor growth in both murine B16 melanoma and human xenograft nasopharyngeal carcinoma (NPC) models (Shen et al. 2013). Another remarkable research of antiangiogenesis was proposed by Yang and his colleagues. They designed a latent membrane protein 1
(LMP1)-targeted DNAzyme (DZ1) to interfere with the tube formation ability of the endothelial cell and downregulated the secretion of VEGF of tumor cells. In vivo experiments, results showed that the DNAzyme significantly inhibited the growth of tumors and reduced the permeability of tumor vessels (Yang et al. 2015). Researches have shown that matrix metalloproteinase-9 (MMP-9) is involved in multiple tumor events such as angiogenesis, the proliferation, and metastasis of tumor cells (Egeblad and Werb 2002). Hallett et al. used an anti-MMP-9 DNAzyme (AM9D) for breast cancer treatment. AM9D inhibited the invasion of breast cancer cells and reduced the volume of the tumor. Moreover, AM9D reduced the vascularization of tumors and induced more apoptosis of tumor cells compared with the untreated group (Hallett et al. 2013). Although DNAzyme-based gene therapy for antiangiogenesis is at the preliminary stage of experimental research, the results are encouraging.

\section{CONCLUSION AND PROSPECTS}

RNA-cleaving DNAzymes have been considered as promising therapeutic reagents in cancer treatment due to their excellent properties, which include easy synthesis and modification, high stability, high cleavage specificity, and catalytic activity. However, inefficient delivery hinders the widespread application of DNAzymes in cancer therapy. Therefore, it is a big challenge for DNAzyme to transport from the administration site to blood circulation, go through the tumor stroma and specifically recognize the cancer cells. For the past few years, remarkable progress has been made in the development of DNAzyme-based nanotherapeutic platforms for cancer gene therapy, which brings hope for the clinical application of these DNAzymes. In this review, we briefly discussed the DNAzyme-based nanotherapeutic platform as promising gene therapy approaches in cancer therapy, including oncogene antagonism therapy, treatment resistance gene therapy, immunogene therapy, and antiangiogenesis gene therapy. New exciting researches for DNAzyme-based gene suppression of adjuvant cancer therapy are underway. First, we would like to point out that more focus could be paid on developing self-supported multifunctional nano-delivery systems to promote the delivery of DNAzymes for enhancing the antitumor therapeutic efficacy. Second, immunotherapy has been demonstrated to an effective therapeutic strategy to combat with cancer. However, only a few successful examples that employed DNAzymes to enhance the overall immunotherapy outcome. 
Therefore, more attention could be paid to silence immunosuppression genes using DNAzymes for amplifying the immunotherapy effect. In addition, the biosafety of the DNAzymes or the nanocarriers should be put into consideration in further studies. Although the DNAzyme-based nanotherapeutic platform is still in its infancy as a cancer gene therapy approach, the development of multifunctional nanocarriers for DNAzyme delivery and cancer gene therapy is likely to open new avenues for cancer therapy in the future.

Acknowledgements The work was supported by the National Natural Science Foundation of China (21603051), the Natural Science Foundation of Hebei Province (B2018201221, B2018201157), Hebei Youth top talent project, Key Projects of Education Department of Hebei Province (ZD2018036), and Undergraduate Innovation and Entrepreneurship Training Program of Hebei University (2020351).

\section{Compliance with Ethical Standards}

Conflict of interest Wendi Huo, Xiaona Li, Bei Wang, Haoran Zhang, Jinchao Zhang, Xinjian Yang, and Yi Jin declare that they have no conflict of interest.

Human and animal rights and informed consent This article does not contain any studies with human or animal subjects performed by any of the authors.

Open Access This article is licensed under a Creative Commons Attribution 4.0 International License, which permits use, sharing, adaptation, distribution and reproduction in any medium or format, as long as you give appropriate credit to the original author(s) and the source, provide a link to the Creative Commons licence, and indicate if changes were made. The images or other third party material in this article are included in the article's Creative Commons licence, unless indicated otherwise in a credit line to the material. If material is not included in the article's Creative Commons licence and your intended use is not permitted by statutory regulation or exceeds the permitted use, you will need to obtain permission directly from the copyright holder. To view a copy of this licence, visit http://creativecommons.org/ licenses/by/4.0/.

\section{References}

Amundson SA, Myers TG, Scudiero D, Kitada S, Reed JC, Fornace AJ Jr (2000) An informatics approach identifying markers of chemosensitivity in human cancer cell Lines. Cancer Res 60(21):6101-6110

Cai H, Cho EA, Li Y, Sockler J, Parish CR, Chong BH, Edwards J, Dodds TJ, Ferguson PM, Wilmott JS, Scolyer RA, Halliday GM, Khachigian LM (2018) Melanoma protective antitumor immunity activated by catalytic DNA. Oncogene 37(37):5115-5126

Cai H, Fernando SS, Leonel P, Wang B, Margaret P, Miles PD, Ghassan JM, Roland S, Christopher RP, Beng HC, Graham JL, Tak-Wah W, Colin NC, Douglas JF, Fergal JM, Ross SB, Halliday GM, Levon MK (2012) DNAzyme targeting c-jun suppresses skin cancer growth. Sci Transl Med 4(139):139ra82-139ra82
Cao T, Wang Y, Tao Y, Zhang L, Zhou YL, Zhang XX, Heyman JA, Weitz DA (2020) DNAzyme-powered nucleic acid release from solid supports. Chem Commun (Camb) 56(4):647-650

Cho E-A, Moloney FJ, Cai H, Au-Yeung A, China C, Scolyer RA, Yosufi B, Raftery MJ, Deng JZ, Morton SW, Hammond PT, Arkenau H-T, Damian DL, Francis DJ, Chesterman CN, Barnetson RSC, Halliday GM, Khachigian LM (2013) Safety and tolerability of an intratumorally injected DNAzyme, Dz13, in patients with nodular basal-cell carcinoma: a phase 1 first-in-human trial (DISCOVER). The Lancet 381(9880):1835-1843

Cully M (2017) Cancer: tumour vessel normalization takes centre stage. Nat Rev Drug Discov 16(2):87

Dass CR, Choong PF, Khachigian LM (2008a) DNAzyme technology and cancer therapy: cleave and let die. Mol Cancer Ther $7(2): 243-251$

Dass CR, Khachigian LM, Choong PF (2008b) c-Jun knockdown sensitizes osteosarcoma to doxorubicin. Mol Cancer Ther 7(7):1909-1912

Davis ME, Chen ZG, Shin DM (2008) Nanoparticle therapeutics: an emerging treatment modality for cancer. Nat Rev Drug Discov 7(9):771-782

Egeblad M, Werb Z (2002) New functions for the matrix metalloproteinases in cancer progression. Nat Rev Cancer 2(3):161-174

Fan H, Zhao Z, Yan G, Zhang X, Yang C, Meng H, Chen Z, Liu H, Tan $\mathrm{W}$ (2015) A smart DNAzyme- $\mathrm{MnO}_{2}$ nanosystem for efficient gene silencing. Angew Chem Int Ed Engl 54(16):4801-4805

Farrokhi F, Karami Z, Esmaeili-Mahani S, Heydari A (2018) Delivery of DNAzyme targeting c-Myc gene using $\beta$-cyclodextrin polymer nanocarrier for therapeutic application in human breast cancer cell line. J Drug Deliv Sci Tec 47:477-484

Feng J, Xu Z, Liu F, Zhao Y, Yu W, Pan M, Wang F, Liu X (2018) Versatile catalytic deoxyribozyme vehicles for multimodal imaging-guided efficient gene regulation and photothermal therapy. ACS Nano 12(12):12888-12901

Fokina AA, Meschaninova MI, Durfort T, Venyaminova AG, Francois JC (2012) Targeting insulin-like growth factor I with 10-23 DNAzymes: 2'-0-methyl modifications in the catalytic core enhance mRNA cleavage. Biochemistry 51(11):2181-2191

$\mathrm{Fu}$ S, Sun L (2015) DNAzyme-based therapeutics for cancer treatment. Future Med Chem 7(13):1701-1707

Gao P, Zhou G, Zhang Q Li H, Mu K, Yuan Y, Zhang J, Wang B (2006) Reversal MDR in breast carcinoma cells by transfection of ribozyme designed according the secondary structure of mdr1 mRNA. Chinese J Physiol 49(2):96-103

Goel HL, Mercurio AM (2013) VEGF targets the tumour cell. Nat Rev Cancer 13(12):871-882

Haabeth OA, Tveita AA, Fauskanger M, Schjesvold F, Lorvik KB, Hofgaard PO, Omholt H, Munthe LA, Dembic Z, Corthay A, Bogen B (2014) How do $\mathrm{CD}^{+}{ }^{+} \mathrm{T}$ cells detect and eliminate tumor cells that either lack or express MHC class II molecules? Front Immunol 5(5):174. https://doi.org/10. 3389/fimmu.2014.00174

Hallett MA, Teng B, Hasegawa H, Schwab LP, Seagroves TN, Pourmotabbed $T$ (2013) Anti-matrix metalloproteinase-9 DNAzyme decreases tumor growth in the MMTV-PyMT mouse model of breast cancer. Breast Cancer Res 15(1):R12. https://doi.org/10.1186/bcr3385

He ZM, Zhang PH, Li X, Zhang JR, Zhu JJ (2016) A Targeted DNAzyme-nanocomposite probe equipped with built-in $\mathrm{Zn}^{2+}$ arsenal for combined treatment of gene regulation and drug delivery. Sci Rep 6:22737. https://doi.org/10.1038/ srep22737

Hollenstein M (2015) DNA catalysis: the chemical repertoire of DNAzymes. Molecules 20(11):20777-20804 
Jang D, Baek YM, Park H, Hwang YE, Kim DE (2018) Dual effects of a CpG-DNAzyme targeting mutant EGFR transcripts in lung cancer cells: TLR9 activation and EGFR downregulation. BMB Rep 51(1):27-32

Jin Y, Li Z, Liu H, Chen S, Wang F, Wang L, Li N, Ge K, Yang X, Liang X-J, Zhang J (2017) Biodegradable, multifunctional DNAzyme nanoflowers for enhanced cancer therapy. NPG Asia Mater 9(3):e365

Jin Y, Liang L, Sun X, Yu G, Chen S, Shi S, Liu H, Li Z, Ge K, Liu D, Yang X, Zhang J (2018) Deoxyribozyme-nanosponges for improved photothermal therapy by overcoming thermoresistance. NPG Asia Mater 10:373-384

Jin Y, Wang H, Li X, Zhu H, Sun D, Sun X, Liu H, Zhang Z, Cao L, Gao C, Wang H, Liang XJ, Zhang J, Yang X (2020) Multifunctional DNA polymer-assisted upconversion therapeutic nanoplatform for enhanced photodynamic therapy. ACS Appl Mater Inter 12(24):26832-26841

Khachigian LM (2019) Deoxyribozymes as catalytic nanotherapeutic agents. Cancer Res 79(5):879-888

Khachigian LM, Cai H, Moloney FJ, Parish CR, Chong BH, Stocker R, Barnetson RS, Halliday GM (2012) Destroying c-jun messenger: new insights into biological mechanisms of DNAzyme function. Oncotarget 3(6):594-595

Lan T, Furuya K, Lu Y (2010) A highly selective lead sensor based on a classic lead DNAzyme. Chem Commun (Camb) 46(22):3896-3898

Li D, Mo F, Wu J, Huang Y, Zhou H, Ding S, Chen W (2018) A multifunctional DNA nano-scorpion for highly efficient targeted delivery of mRNA therapeutics. Sci Rep 8(1):10196. https://doi.org/10.1038/s41598-018-28542-3

Liu J, Li Y (2007) A DNAzyme catalytic beacon sensor for paramagnetic $\mathrm{Cu}^{2+}$ ions in aqueous solution with high sensitivity and selectivity. J Am Chem Soc 129(32):9838-9839

Lugano R, Ramachandran M, Dimberg A (2019) Tumor angiogenesis: causes, consequences, challenges and opportunities. Cell Mol Life Sci 77(21):1745-1770

Maeda H, Wu J, Sawa T, Matsumura Y, Hori K (2000) Tumor vascular permeability and the EPR effect in macromolecular therapeutics: a review. J Control Release 65(1-2):271-284

Meng L, Ma W, Lin S, Shi S, Li Y, Lin Y (2019) Tetrahedral DNA nanostructure-delivered DNAzyme for gene silencing to suppress cell growth. ACS Appl Mater Inter 11(7):6850-6857

Peng S, Bie B, Sun Y, Liu M, Cong H, Zhou W, Xia Y, Tang H, Deng H, Zhou X (2018) Metal-organic frameworks for precise inclusion of single-stranded DNA and transfection in immune cells. Nat Commun 9(1):1293. https://doi.org/10.1038/s41467018-03650-w

Peng Y, Zhang P, Huang X, Yan Q, Wu M, Xie R, Wu Y, Zhang M, Nan Q Zhao J, Li A, Xiong J, Ren Y, Bai Y, Chen Y, Liu S (2016) Direct regulation of foxk1 by c-jun promotes proliferation, invasion and metastasis in gastric cancer cells. Cell Death Dis 7(11):e2480. https://doi.org/10.1038/cddis.2016.225

Ramos P, Bentires-Alj M (2015) Mechanism-based cancer therapy: resistance to therapy, therapy for resistance. Oncogene 34(28):3617-3626

Raphael I, Joern RR, Forsthuber TG (2020) Memory CD4 ${ }^{+}$T cells in immunity and autoimmune diseases. Cells 9(3):531. https://doi.org/10.3390/cells9030531

Ren T, Deng Z, Liu H, Li X, Li J, Yuan J, He Y, Liu Q, Yang Y, Zhong S (2019) Co-delivery of DNAzyme and a chemotherapy drug using a DNA tetrahedron for enhanced anticancer therapy through synergistic effects. New J Chem 43(15):14020-14027

Roma-Rodrigues C, Rivas-Garcia L, Baptista PV, Fernandes AR (2020) Gene therapy in cancer treatment: why go nano?
Pharmaceutics 12(3):233. https://doi.org/10.3390/ pharmaceutics12030233

Santoro SW, Joyce GF (1997) A general purpose RNA-cleaving DNA enzyme. Proc Natl Acad Sci USA 94(9):4262-4266

Shen L, Zhou Q, Wang Y, Liao W, Chen Y, Xu Z, Yang L, Sun LQ (2013) Antiangiogenic and antitumoral effects mediated by a vascular endothelial growth factor receptor 1 (VEGFR-1)targeted DNAzyme. Mol Med 19(1):377-386

Silverman SK (2005) In vitro selection, characterization, and application of deoxyribozymes that cleave RNA. Nucleic Acids Res 33(19):6151-6163

Silverman SK (2016) Catalytic DNA: scope, applications, and biochemistry of deoxyribozymes. Trends Biochem Sci 41(7):595-609

Sun SP, Liu CP, Huang IP, Chu CH, Chung MF, Cheng SH, Lin SY, Lo LW (2017) A co-delivery nanosystem of chemotherapeutics and DNAzyme overcomes cancer drug resistance and metastasis. Nano Futures 1(3):035005. https://doi.org/10.1088/ 2399-1984/aa996f

Sun X, Jin Y, Wang H, Feng N, Li Z, Liu D, Ge K, Liu H, Zhang J, Yang $X$ (2018) A NIR-light activated nanoplatform for sensitizing triple negative breast cancer against therapeutic resistance to enhance the treatment effect. J Mater Chem B 6(43):6950-6956

Tan ML, Dunstan DE, Friedhuber AM, Choong PF, Dass CR (2010) A nanoparticulate system that enhances the efficacy of the tumoricide Dz13 when administered proximal to the lesion site. J Control Release 144(2):196-202

Wang H, Chen Y, Wang H, Liu X, Zhou X, Wang F (2019a) DNAzyme-loaded metal-organic frameworks (MOFs) for selfsufficient gene therapy. Angew Chem Int Ed Engl 58(22):7380-7384

Wang J, Wang H, Wang H, He S, Li R, Deng Z, Liu X, Wang F (2019b) Nonviolent self-catabolic DNAzyme nanosponges for smart anticancer drug delivery. ACS Nano 13(5):5852-5863

Wang X, Dai J, Wang X, Hu Q Huang K, Zhao Z, Lou X, Xia F (2019c) $\mathrm{MnO}_{2}$-DNAzyme-photosensitizer nanocomposite with AIE characteristic for cell imaging and photodynamic-gene therapy. Talanta 202(1):591-599

Wang X, Zhang L, Ding N, Yang X, Zhang J, He J, Li Z, Sun LQ (2015) Identification and characterization of DNAzymes targeting DNA methyltransferase I for suppressing bladder cancer proliferation. Biochem Biophys Res Commun 461(2):329-333

Wiktorska M, Papiewska-Pająk L, Okruszek A, Sacewicz Hofman L, Niewiarowska J (2010) DNAzyme as an efficient tool to modulate invasiveness of human carcinoma cells. Acta Biochimica Polonica 57(3):269-275

Wu Y, Yu L, Mcmahon R, Rossi J, Forman S, Snyder D (1999) Inhibition of bcr-abl oncogene expression by novel deoxyribozymes (DNAzymes). Hum Gene Ther 10(17):2847-2857

Xiao Y, Shi K, Qu Y, Chu B, Qian Z (2019) Engineering nanoparticles for targeted delivery of nucleic acid therapeutics in tumor. Mol Ther Methods Clin Dev 12:1-18

Xing Z, Gao S, Duan Y, Han H, Li L, Yang Y, Li Q (2015) Delivery of DNAzyme targeting aurora kinase A to inhibit the proliferation and migration of human prostate cancer. Int J Nanomed 10(1):5715-5727

Xu Z, Yang L, Sun L, Cao Y (2012) Use of DNAzymes for cancer research and therapy. Chinese Sci Bull 57(26):3404-3408

Yang L, Liu L, Xu Z, Liao W, Feng D, Dong X, Xu S, Xiao L, Lu J, Luo X, Tang M, Ann MB, Dong Z, Sun L, Cao Y (2015) EBV-LMP1 targeted DNAzyme enhances radiosensitivity by inhibiting tumor angiogenesis via the JNKs/HIF-1 pathway in nasopharyngeal carcinoma. Oncotarget 6(8):5804-5817 
Yu X, Yang L, Cairns MJ, Dass C, Saravolac E, Li X, Sun L (2014) Chemosensitization of solid tumors by inhibition of Bcl-xL expression using DNAzyme. Oncotarget 5(19):9039-9048

Zhang L, Zhao W, Liang C, Yi X, Pei Y, Lin Y, He J, Li W (2016) VEGFR-1 targeted DNAzyme via transcatheter arterial delivery influences tumor vasculature assessed through dynamic contrast-enhanced magnetic resonance imaging. Oncol Rep 36(3):1339-1344

Zhou W, Ding J, Liu J (2017) Theranostic DNAzymes. Theranostics $7(4): 1010-1025$
Zhou W, Saran R, Chen Q Ding J, Liu J (2016) A new $\mathrm{Na}^{+}$. dependent RNA-cleaving DNAzyme with over 1000 -fold rate acceleration by ethanol. Chembiochem 17(2):159-163

Zokaei E, Badoei-Dalfrad A, Ansari M, Karami Z, Eslaminejad T, Nematollahi-Mahani SN (2019) Therapeutic potential of DNAzyme loaded on chitosan/cyclodextrin nanoparticle to recovery of chemosensitivity in the MCF-7 cell line. Appl Biochem Biotechnol 187(3):708-723 